\title{
Dietary intake in relation to clinical status in patients with psoriasis
}

\author{
BY KARI SOLVOLL ${ }^{1}$, ELISABETH SøYLAND ${ }^{2}$, BERIT SANDSTAD ${ }^{1}$ \\ AND CHRISTIAN A. DREVON ${ }^{1}$ \\ ${ }^{1}$ Institute for Nutrition Research, University of Oslo, PO Box 1046 Blindern, N-0316 Oslo, Norway \\ ${ }^{2}$ Department of Dermatology, Rikshospitalet, Pilestredet 32, N-0027 Oslo, Norway
}

(Received 10 July 1995 - Revised 7 May 1996 - Accepted 3 July 1996)

\begin{abstract}
The aim of the present study was to examine whether the composition of the diet among patients with psoriasis differed from a regular Norwegian diet and to relate nutrient intake to the clinical status of the patients' disease. Patients were recruited from all five university Departments of Dermatology in Norway. The participants filled in a quantitative food-frequency questionnaire before attending a clinical trial. Dietary data were available from ninety-three men and forty-three women, aged 17-73 years, with stable plaque psoriasis involving more than $8 \%$ of the body surface. The dietary intake of the patients was compared with the diet of a reference group randomly selected from the Norwegian population, and related to a dermatological score of the psoriatics. The patients' intakes of energy and nutrients were fairly similar to those found in the reference group, except for a lower content of carbohydrates and sugar in the diet of female patients. None of the nutrients gave statistically significant contributions to explain the clinical score values when analysed by correlations.
\end{abstract}

Psoriasis: Nutrient intake

Psoriasis is a skin disease which diminishes the quality of life for a large number of subjects. Approximately $2 \%$ of the population in Western countries is affected by the chronic psoriatic skin disease. The molecular basis for the pathogenesis of psoriasis is unknown. However, there is evidence that the disease is influenced by genetic, inflammatory, immunological and dietary factors (Suarez-Almazor \& Russel, 1990; Barker, 1991; Breathnach, 1993, Søyland \& Drevon, 1993). In recent years, vitamin A and vitamin $\mathrm{D}$ analogues have been successfully used in the topical treatment of patients with psoriasis (Ott \& Geiger, 1983; Smith et al. 1988). Also, dietary supplementation with fish oil has been used in clinical trials among psoriatic patients, but the results are contradictory (Ziboh et al. 1986; Bittiner et al. 1988; Bjørneboe et al. 1988; Søyland et al. 1993a). In a crossover-designed dietary trial among patients with plaque psoriasis in which inclusion of oily fish was compared with that of white fish, a modest improvement was observed in the clinical condition during the period in which oily fish was included in the diet (Collier et al. 1993). Furthermore, it has been reported that high intake of alcohol may be associated with more extensive expression of the disease (Farber \& Nall, 1994). On this basis, we examined the association between the dietary intake of nutrients and the clinical status of psoriatic men and women. Since dietary approaches have been tried by many psoriatic patients, we wanted to test whether their nutrient intake differed from the typical diet among a representative group of the Norwegian population. Thus, we compared the diet of individuals with psoriasis with the diet of a reference group, in particular to determine 
whether the intake of vitamin A, vitamin $\mathrm{D}$ or very-long-chain $n-3$ fatty acids was lower among psoriatic patients than reference subjects.

\section{METHODS}

\section{Patients}

Men and women ( $n$ 145) with stable plaque psoriasis involving more than $8 \%$ of the body surface, were recruited to a clinical trial evaluating the effect of supplementation with verylong-chain $n$-3 fatty acids (Søyland et al. 1993a). The participants were selected among out-patients from five different departments of dermatology at Norwegian University hospitals. Dietary data were available from ninety-three men (mean age 46 (range 17-72) years) and forty-three women (mean age 46 (range 18-73) years).

\section{Dietary monitoring}

Before the start of the study, the patients' usual diet was assessed by a self-administered quantitative food-frequency questionnaire. It comprised approximately 180 food items and was designed to cover the whole diet (Solvoll et al. 1993). The frequency of consumption was given daily, weekly or monthly depending on the food item. The portion sizes were units such as slices, glasses, cups, pieces, decilitres and spoonfuls. For all main-meal food items the frequencies of use ranged from one to thirteen or more per month, and there were five alternative portion sizes. The portion sizes of the different food items were converted to weights on the basis of standard portions estimated from previous Norwegian studies. The accuracy of the questionnaire was tested in a group of elderly women. Unadjusted Spearman rank correlation coefficients comparing intakes measured by the questionnaire and multiple weighed-diet records for a total of $14 \mathrm{~d}$ ranged from 0.31 for vitamin $\mathrm{C}$ to 0.79 for carbohydrates, the median being 0.61 (Nes et al. 1992). An evaluation of the questionnaire was also performed among dermatology out-patients (psoriasis and atopic dermatitis), who filled in the questionnaire and participated in a $48 \mathrm{~h}$ recall interview at the start and at the end of the previously mentioned clinical trial. The Pearson correlation coefficients for energy and sixteen nutrients were on average 0.44 and 0.50 at the two examinations respectively (Solvoll et al. 1993).

In the present study the questionnaire was mailed to the psoriatic patients, filled in at home and brought along to the first physical examination at the hospital. The intakes of energy and nutrients were calculated using a food database developed at the Section for Dietary Research, Institute for Nutrition Research, University of Oslo. Dietary supplements such as cod-liver-oil and fish-oil capsules are included in the nutrient calculations. Data for men and women are presented separately, since the nutritional requirements and food habits are different for the two genders.

The dietary data were compared with results from a nationwide study performed in 1992 in collaboration with Statistics Norway, which was responsible for sampling of participants and collection of the data. The sample ( $n 1200$, response rate $60 \%$ ) was drawn according to procedures for random sampling. This study covered all the different parts of the country, and the same food-frequency questionnaire as that completed by psoriatic patients was used for data collection. The same database was used for calculation of the intake of energy and nutrients for the patients as well as for the reference subjects. Participants aged 15-74 years were chosen to serve as a reference group. This group comprised data for 284 men (mean age 45 years) and 249 women (mean age 41 years). 


\section{Clinical assessment}

As an objective measure of the severity of the illness, the physicians' evaluations at the first examination before start of the trial were used. Erythema, infiltration, desquamation, and the area of skin involved were scored separately for the head, trunk, arms and legs, and these scores were mutliplied by a corresponding factor and summed to produce the score on the psoriasis area and severity index (Fredriksson \& Petterson, 1978). The mean score values were 12.4 (range 4.6-37.2) and 9.9 (range 3.3-22.8) for men and women respectively.

\section{Statistics}

The age distribution in the reference group was somewhat different from the age distribution in the group of psoriatic patients. It was decided, therefore, to age-standardize by the direct method to adjust for the difference in age distributions. The base distribution was that for the Norwegian population of 31 December 1992 (Statistics Norway, 1994). Age intervals of 10 years were chosen for the subjects aged 30-59 years. Those in the agegroup 15-29 years as well as those between 60 and 74 years were pooled due to the small number of patients in these age-groups. Data for the two genders were analysed separately. Age-adjusted means and appropriate standard deviations and mean differences are presented. The two-sample $t$ test was used to determine differences in dietary intake between psoriatic patients and the reference group. Pearson correlation coefficients were calculated to evaluate relationships between dietary intake and score values among men and women with psoriasis.

\section{RESULTS}

\section{Dietary evaluation}

Age-adjusted values for dietary intake for the group of psoriatic men was similar to the intakes of energy and nutrients for the reference group (Table 1). Also, among the psoriatic women, most of the intakes were similar to those found in the reference group (Table 1). However, the percentages of energy derived from carbohydrates and from sugar were lower in psoriatic women, as compared with the reference group $(P=0.042$ and 0.019 , respectively by two-sided $t$ test). The percentages derived from fat and alcohol were higher among the psoriatic women, but these differences were not significant.

The fatty acid pattern in the diet was similar for the two groups of men as well as for the two groups of women (Table 2). The $20: 5 n-3: 20: 4 n-6$ value was highest for the patients, but none of the differences were significant $(P=0.39$ and $P=0.07$ for men and women respectively).

\section{Clinical evaluation}

A possible relationship between dietary intake and clinical status was evaluated by calculating the correlation coefficients between dietary intake and the score value. Plots for the intakes of fish and vitamin D are shown for the group of male patients (Fig. 1). Neither this, nor any of the other foods or nutrients in the diet were significantly correlated with the score values. 


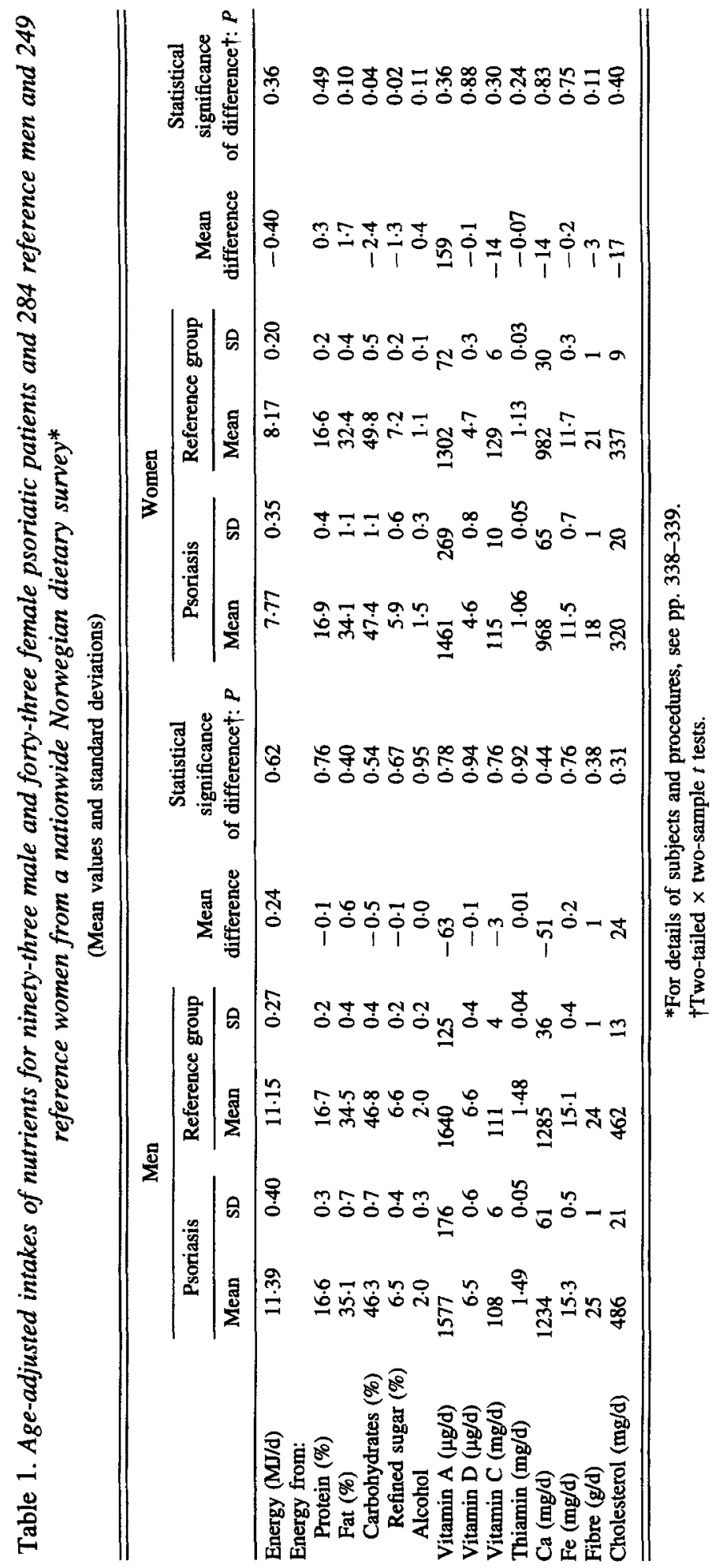


DIET AND PSORIASIS

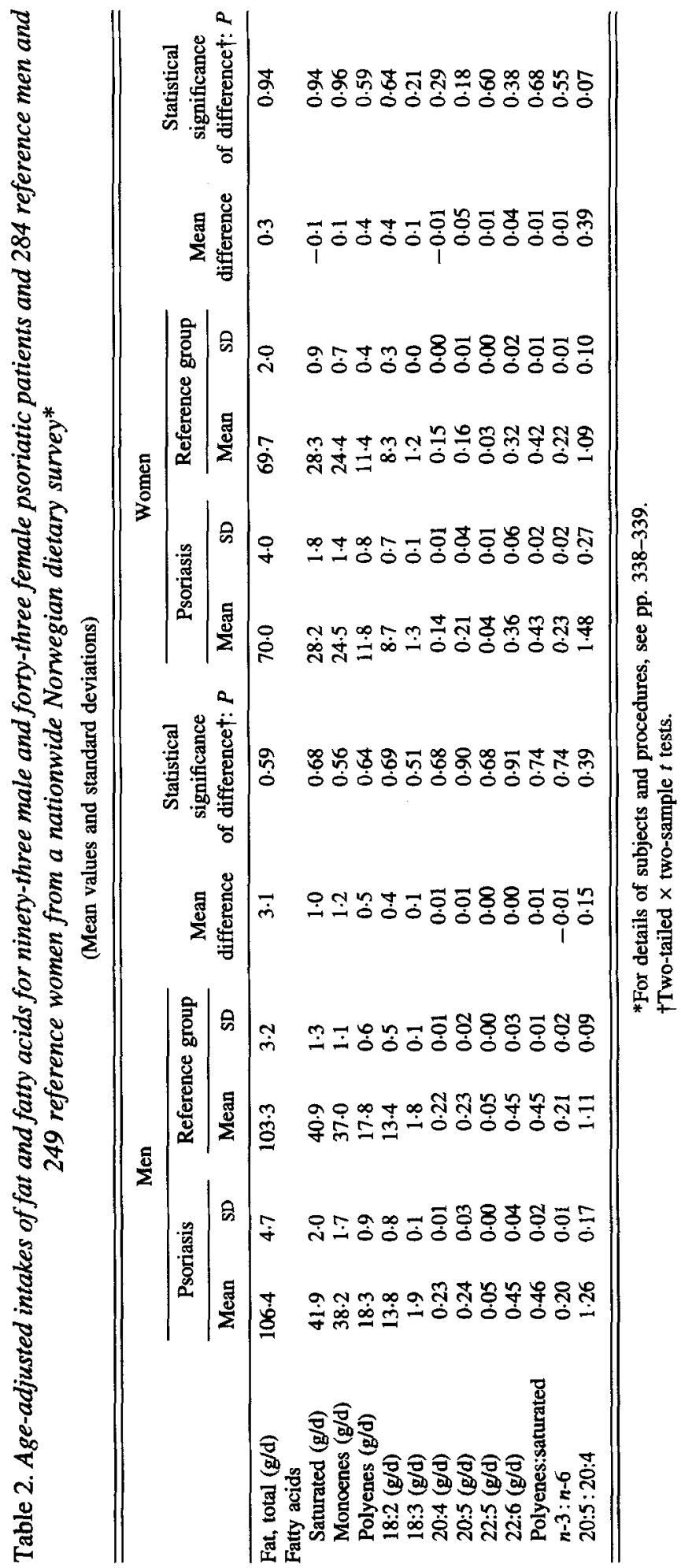



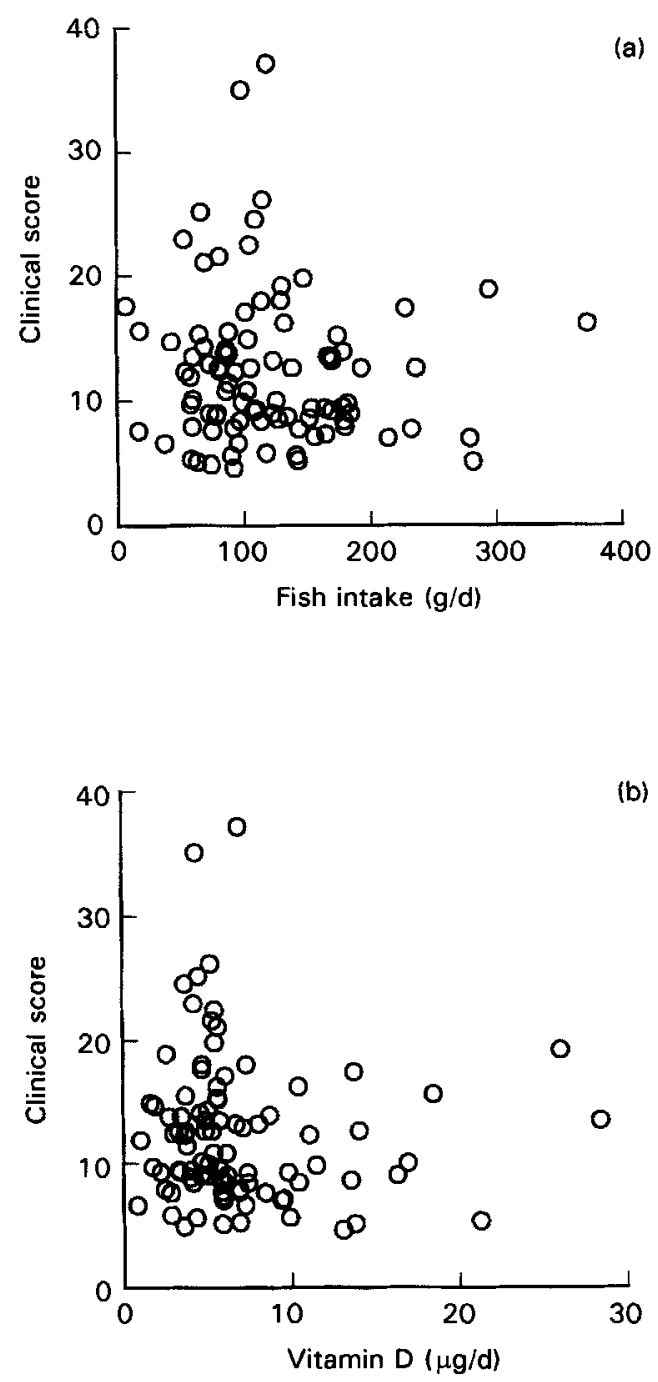

Fig. '1. Scatter plots of the relationship between (a) intake of fish and clinical score $(r-0.070)$ and (b) dietary intake of vitamin D and clinical score $(r-0.071)$ for a group of Norwegian psoriatic men. For details of subjects and procedures, see pp. 338-339

\section{DISCUSSION}

The composition of the diet for our group of psoriatic out-patients was fairly similar to the diet of the reference group. The only significant difference we found was for psoriatic women who had a lower content of carbohydrates and sugar in their diets than women in the reference group.

The accuracy of the results will be influenced by the validity of the dietary data. Dietary intake is difficult to measure precisely, and all survey methods have limitations, including the food-frequency questionnaire (Briefel et al. 1992). However, a validation study applied in a group of elderly women showed good accordance between our questionnaire and $14 \mathrm{~d}$ of weighed records (Nes et al. 1992). Also, an evaluation of results 
from the questionnaire and $48 \mathrm{~h}$-recall interviews among patients with psoriasis and atopic dermatitis (including some of the presently described patients) showed that the questionnaire was a valuable tool for categorizing individuals according to their dietary intake (Solvoll et al. 1993).

The number of participants in our trial was high compared with many other studies among patients with psoriasis. It is not clear whether the dietary data from our study were representative of patients with plaque psoriasis in general. Patients who are willing and able to participate in a clinical trial for several months may be a selected group.

We related the dietary intake to the clinical status of the patients, but none of the nutrients gave statistically significant contribution to explain the score values when analysed by correlations.

In contrast to what has been found previously (Poikolainen et al. 1994) we did not find higher intake of alcohol among patients than among participants in the reference group.

In several studies, dietary fish oil has been reported to have beneficial effects on psoriasis. However, the results are contradictory. With one exception only (Søyland et al. $1993 a$ ) these results are based on open studies and/or inclusion of a small number of patients (Ziboh et al. 1986; Bittiner et al. 1988; Bjørneboe et al. 1988). Very-long-chain $n$ 3 fatty acids (20:5 and 22:6) found in fish oil have anti-proliferative effect on T cells in vitro (Søyland et al. 1993b). Furthermore, eicosapentaenoic acid is the precursor of leukotriene (LT) $B_{5}$, a less chemotatic agent than LTB $_{4}$ which is found to be elevated in skin with psoriatic plaques (Lee et al. 1985). In our multi-centre study, dietary supplementation with very-long-chain $n-3$ fatty acids was no better than maize oil in treating psoriasis, even though there was a marked increase in these very-long-chain fatty acids in serum phospholipids in the experimental group, as compared with the maize oil group (Søyland et al. 1993a).

In a crossover-designed trial published in the United Kingdom, modest clinical improvement of plaque psoriasis was obtained when the participants replaced intake of $170 \mathrm{~g}$ white fish daily with the same amount of oily fish for a period of 6 weeks (Collier $e t$ al. 1993). Oily fish is rich in very-long-chain $n-3$ fatty acids as well as in vitamin $D$. It could be expected that the patients had been conscious of these nutrients in the hope of relieving the symptoms of the disease and, therefore, had consumed more oily fish and/or cod liver than the reference subjects. Of the male and female patients 24 and $16 \%$ respectively reported use of cod-liver oil. However, the frequency of use varied markedly, from very seldom to daily use, and the range of consumption was from 0.1 to $11.0 \mathrm{~g} / \mathrm{d}$. Those using the highest amount gained a considerable supplement of vitamin $A$ and $D$, as well as the very-long-chain $n-3$ fatty acids. In the reference groups $24 \%$ reported use of cod-liver oil.

We have not evaluated the exposure to sunlight or measured the concentration of vitamin $\mathrm{D}$ metabolites in plasma in our patients. Thus, we do not know the importance of the total available vitamin $D$ for the clinical expression of psoriasis. It should be noted that the participants were included during the months November to January.

In summary, the diet among psoriatic out-patients did not differ from what was found in a reference group, except for a lower content of carbohydrates and sugar in the diet of female patients. None of the nutrients gave statistically significant contributions to explain the clinical score values when analysed by correlations.

\section{REFERENCES}

Barker, J. N. W. N. (1991). The pathophysiology of psoriasis. Lancet 338, 227-230.

Bittiner, S. B., Tucker, W. F. G., Cartwright, I. \& Bleehen, S. S. (1988). A double-blind, randomised, placebocontrolled trial of fish oil in psoriasis. Lancet i, 378-380. 
Bjømeboe, A., Smith, A. K., Bjørneboe, G.-E. A., Thune, P. O. \& Drevon, C. A. (1988). Effect of dietary supplementation with $n-3$ fatty acids on clinical manifestations of psoriasis. British Journal of Dermatology 118, 77-83.

Breathnach, S. M. (1993). The skin immune system and psoriasis. Clinical and Experimental Immunology 91, 1343-1345.

Briefel, R. R., Flegal, K. M., Winn, D. M., Loria, C. M., Johnson, C. L. \& Sempos, C. T. (1992). Assessing the nation's diet: limitations of the food frequency questionnaire. Joumal of the American Dietetic Association 92, 959-962.

Collier, P. M., Ursell, A., Zaremba, K., Rowland Payne, C. M. E., Staughton, R. C. D. \& Sanders, T. (1993). Effect of regular consumption of oily fish compared with white fish on chronic plaque psoriasis. European Journal of Clinical Nutrition 47, 251-254.

Farber, E. M. \& Nall, L. (1994). Psoriasis and alcoholism. Cutis 53, 21-27.

Fredriksson, T. \& Petterson, U. (1978). Severe psoriasis - oral therapy with a new retinoid. Dermatologica 157, 238-244.

Lee, T. H., Hoover, R. L., Williams, J. D., Sperling, R. I., Ravalese, J, III, Spur, B. W., Robinson, D. R., Corey, E. J., Lewis, R. A. \& Austen, K. F. (1985). Effect of dietary enrichment with eicosapentaenoic and docosahexaenoic acids on in vitro neutrophil and monocyte leukotriene generation and neutrophil function. New England Journal of Medicine 312, 1217-1224.

Nes, M., Andersen, L. F., Solvoll, K., Sandstad, B., Hustvedt, B. E., Løvø, A. \& Drevon, C. A. (1992). Accuracy of a quantitative food frequency questionnaire applied in elderly Norwegian women. European Journal of Clinical Nutrition 46, 809-821.

Ott, F. \& Geiger, J. (1983). Therapeutic effect of a retinoid Ro 13-6298 in psoriasis. Archives for Dermatological Research 275, 257-258.

Poikolainen, K., Reunala T. \& Karvonen, J. (1994). Smoking, alcohol and life events related to psoriasis among men. British Journal of Dermatology 130, 473-477.

Smith, E., Pincus, S.H., Donovan, L. \& Holick, M. F. (1988). A novel approach for the evaluation and treatment of psoriasis. Oral and topical use of 1,25-dihydroxyvitamin D3 can be a safe and effective therapy of psoriasis. Journal of the American Academy of Dermatology 19, 516-528.

Solvoll, K., Lund-Larsen, K., Søyland, E., Sandstad, B. \& Drevon, C. A. (1993). A quantitative food frequency questionnaire evaluated in a group of dermatologic outpatients. Scandinavian Journal of Nutrition 37, 150155.

Statistics Norway (1994). Statistical Yearbook 1994, 113th ed. Oslo: Kongsvinger.

Suarez-Almazor, M. E. \& Russel, A. S. (1990). The genetics of psoriasis: haplotype sharing in siblings with the disease. Archives of Dermatology 126, 1040-1042.

Søyland, E. \& Drevon, C. A. (1993). The effect of very long-chain $n-3$ fatty acids on immune-related skin diseases. European Journal of Clinical Nutrition 47, 381-388.

Søyland, E., Funk, J., Rajka, G., Sandberg, M., Thune, P., Rustad, L., Helland, S., Middelfart, K., Odu, S., Falk, E. S., Solvoll, K., Bjømeboe, G.-E. \& Drevon, C. A. (1993a). Effect of dietary supplementation with very long-chain $n$-3 fatty acids in patients with psoriasis. New England Journal of Medicine 328, 1812-1816.

Søyland, E., Nenseter, M. S., Braathen, L. R. \& Drevon, C. A. (1993b). Very long-chain $n-3$ and $n-6$ polyunsaturated fatty acids inhibit proliferation of human $\mathrm{T}$ lymphocytes in vitro. European Journal of Clinical Investigation 23, 112-121.

Ziboh, V. A., Cohen, K. A., Ellis, C. N., Miller, C., Hamilton, T. A., Kragballe, K., Hydrich, C. R. \& Voorhees, J. J. (1986). Effects of dietary supplementation of fish oil on neutrophil and epidermal fatty acids. Archives of Dermatology 122, 1277-1282. 